Чиркунов Ю. А. Новосибирский государственный архитектурно-строительный университет (Сибстрин), г. Новосибирск, Россия

chr101@mail.ru

\title{
АВТОМОДЕЛЬНЫЕ ВОЛНЫ ТРЕХМЕРНОЙ МОДЕЛИ ВЕСТЕРВЕЛЬТА ПРИ НАЛИЧИИ ДИССИПАЦИИ
}

Введение:

Многие математические модели механики сплошной среды сформулированы в виде линейных и квазилинейных дифференциальных уравнений. Симметричный анализ этих моделей является одним из наиболее эффективных способов получения количественных и качественных характеристик описываемых ими физических процессов. Актуальность исследования модели Вестервельта обусловлена использованием этой модели для расчета параметрических антенн сонаров и для расчета ультразвуковых полей в медицине.

Материалы и методы исследований:

Методами исследований являются: групповой (симметрийный) анализ дифференциальных уравнений и общие методы математической физики.

Результаты исследований

и их обсуждение: Исследуется важная для приложений трехмерная модель Вестервельта нелинейной гидроакустики при наличии диссипации. Установлено, что среди инвариантных подмоделей ранга 1 эта модель имеет только три типа существенно различных (не связанных точечными преобразованиями) автомодельных волн. К ним относятся: автомодельная волна, распространяющаяся вдоль одной из осей координат, плоская автомодельная круговая волна и автомодельная сферически-симметричная волна. Получены интегро-дифференциальные уравнения, описывающие эти автомодельные волны. При некоторых условиях установлены существование и единственность таких автомодельных волн.

Выводы: $\quad$ В нелинейной гидроакустике модель Вестервельта используется для исследования ультразвуковых полей, генерируемых мощными излучателями. Для трехмерной модели Вестервельта при наличии диссипации установлено, что среди инвариантных подмоделей ранга 1 эта модель имеет только три типа существенно различных (не связанных точечными преобразованиями) автомодельных волн. Исследованы эти волны. Наличие произвольных постоянных в полученных интегро-дифференциальных уравнениях, описывающих эти автомодельные волны открывает новые возможности для изучения других (отличных от исследованных в статье) имеющих физический смысл краевых задач. Практическая значимость этого исследования обусловлена использованием модели Вестервельта для расчета параметрических антенн сонаров и для расчета ультразвуковых полей в медицине.

Ключевые слова: нелинейная гидроакустика, модель Вестервельта при наличии диссипации, автомодельные волны. 
Chirkunov Yu. A. Novosibirsk state University of architecture and civil engineering, Novosibirsk, Russia

\section{SELF-SIMILAR WAVES OF THREE- DIMENSIONAL MODEL OF WESTERVELT IN THE EXISTENCE OF DISSIPATION}

Introduction: Many mathematical models of continuum mechanics are formulated as linear and quasilinear differential equations. Symmetric analysis of these models is one of the most effective ways to obtain the quantitative and qualitative characteristics of the physical processes described by them. The relevance of the study of the Westervelt model is due to the use of this model for calculating parametric sonar antennas and for calculating ultrasonic fields in medicine

Materials and

research methods: Research methods are: group (symmetry) analysis of differential equations and general methods of mathematical physics.

Research results

and their discussion: The three-dimensional Westervelt model of nonlinear hydroacoustics, which is important for applications, is investigated in the presence of dissipation. It has been established that among the invariant submodels of rank 1, this model has only three types of essentially different (not connected by point transformations) self-similar waves. These include the self-similar wave propagating along one of the axes of coordinates, the flat self-similar circular wave and the self-similar spherically symmetric wave. Integro-differential equations describing these self-similar waves are obtained. Under some conditions, the existence and uniqueness of such self-similar waves are established.

Conclusions: In nonlinear hydroacoustics, the Westervelt model is used to study ultrasonic fields generated by high-power radiators. For the Westervelt three-dimensional model in the presence of dissipation, it was found that among the invariant submodels of rank 1 this model has only three types of essentially different (not connected by point transformations) selfsimilar waves. These waves are investigated. The presence of arbitrary constants in the obtained integro-differential equations describing these self-similar waves opens up new possibilities for studying other boundary value problems (other than those studied in the article) that have a physical meaning. The practical significance of this study is due to the use of the Westervelt model for calculating parametric sonar antennas and for calculating ultrasonic fields in medicine.

Keywords: nonlinear underwater acoustics, Westervelt model in the presence of dissipation, self-similar waves. 


\section{Введение}

Многие математические модели механики сплошной среды сформулированы в виде линейных и квазилинейных дифференциальных уравнений. При выводе этих уравнений использована их инвариантность при различных преобразованиях пространства-времени. Но симметрии модели могут быть скрыты, они могут быть результатом физических свойств моделируемого явления. Использование всех свойств симметрии позволяет корректно моделировать явление и классифицировать подмодели. Симметричный анализ этих моделей является одним из наиболее эффективных способов получения количественных и качественных характеристик описываемых ими физических процессов.

В нелинейной гидроакустике модель Вестервельта [1 - 11] используется для исследования ультразвуковых полей, генерируемых мощными излучателями. В этой модели не делается предположения о малости углов дифракции, что позволяет более точно учитывать дифракционные эффекты. Наиболее известными практическими применениями модели Вестервельта являются использование этой модели для расчета параметрических антенн сонаров и для расчета ультразвуковых полей в медицине.

В настоящей работе исследуется модель Вестервельта при наличии диссипации. Эта модель задается следующим уравнением Вестервельта [1 10]:

$$
P_{x x}+P_{y y}+P_{z z}-\frac{1}{c_{0}^{2}} P_{T T}+\frac{\varepsilon}{\rho_{0} c_{0}^{4}}\left(P^{2}\right)_{T T}+\frac{b}{\rho_{0} c_{0}^{4}} P_{T T T}=0
$$

$P=(T, x)-$ акустическое давление,

$T-\quad$ время,

$x=(x, y, z) \in R^{3}$.

$\varepsilon-\quad$ параметр нелинейности,

$\rho_{0}-\quad$ равновесная плотность среды,

$c_{0}-\quad$ скорость звука.

С помощью переменных

$$
t=c_{0} T, \quad p(t, \boldsymbol{x})=\frac{\varepsilon}{\rho_{0} c_{0}^{2}} P(T, \boldsymbol{x})
$$

уравнение (1) записывается в следующем виде

$$
p_{x x}+p_{y y}+p_{z z}-p_{t t}+\left(p^{2}\right)_{t t}+p_{t t t}=0
$$

Уравнение (3) является основным объектом дальнейшего исследования. 


\section{Материалы и методы исследований}

В настоящей работе исследуются автомодельные волны трехмерной модели Вестервельта нелинейной гидроакустики при наличии диссипации. Получены три типа существенно различных (не связанных точечными преобразованиями) автомодельных волн. К ним относятся: автомодельная волна, распространяющаяся вдоль одной из осей координат, плоская автомодельная круговая волна и автомодельная сферически-симметричная волна. Получены интегро-дифференциальные уравнения, описывающие эти автомодельные волны. При некоторых условиях установлены существование и единственность таких автомодельных волн. Методами исследований являются: групповой (симметрийный) анализ дифференциальных уравнений [12] и общие методы математической физики.

\section{Результаты исследований и их обсуждение}

1. Групповое свойство

Основная группа Ли преобразований уравнения (3) определяется по стандартному алгорипму [12] и порождается операторами:

$$
\begin{aligned}
& X_{0}=\partial_{t}, \quad X_{1}=\partial_{x}, \quad X_{2}=\partial_{y}, \quad X_{3}=\partial_{z}, \\
& R=2 t \partial_{t}+3 x \partial_{x}+3 y \partial_{y}+3 z \partial_{z}-(2 p-1) \partial_{p}, \\
& Z_{1}=y \partial_{z}-z \partial_{y}, \quad Z_{2}=z \partial_{x}-x \partial_{z}, \quad Z_{3}=x \partial_{y}-y \partial_{x},
\end{aligned}
$$

Алгебра Ли с базисом (4) является прямой суммой фактора Леви с базисом

\section{$Z_{1}, Z_{2}, Z_{3}$ и ее радикала с базисом $X_{0}, X_{1}, X_{2}, X_{3}, R$.}

Инвариантные подмодели ранга 1 задаются инвариантными решениями ранга 1 уравнения (3). Действие группы внутренних автоморфизмов основной алгебры Ли (4) уравнения (3) на эту алгебру разбивает ее на непересекающиеся классы подобных подалгебр. Выбор простейшего представителя в каждом классе дает оптимальную систему неподобных подалгебр основной алгебры Ли этого уравнения. Каждой подалгебре этой оптимальной системы соответствует подгруппа основной группы уравнения (3), порожденная этой подалгеброй. Из необходимых условий существования инвариантных решений [12] следует, что все не связанные точечными преобразованиями инвариантные решения ранга 1 уравнения (3), суть решения, инвариантные относительно некоторых трехпараметрических и четырехпараметрических подгрупп основной группы уравнения (3). Автомодельные волны описываются решениями, инвариантными относительно подгрупп, базис алгебры Ли которых не содержит оператор переноса по времени $X_{0}$ и содержит оператор, зависящий от оператора растяжения $R$. Действие группы внутрен- 
них автоморфизмов основной алгебры Ли (4) на эту алгебру показывает, что множество таких трехпараметрических и четырехпараметрических подгрупп с точностью до преобразований группы внутренних автоморфизмов основной алгебры Ли (4) исчерпывается следующими подгруппами:

$$
\begin{aligned}
& R+\alpha Z_{3}, X_{1}, X_{2}, \\
& R, X_{3}, Z_{3}, \\
& R, X_{1}, X_{2}, Z_{3}, \\
& R, Z_{1}, Z_{2}, Z_{3} .
\end{aligned}
$$

где $\quad \alpha-\quad$ произвольная вещественная постоянная.

\section{2. Автомодельные волны}

Применение критерия инвариантности функции относительно группы Ли преобразований [12] дает универсальный инвариант каждой подгруппы оптимальной системы подгрупп в пространстве $R^{5}(t, x, p)$. Знание универсального инварианта подгруппы позволяет получить общий вид решения, инвариантного относительно этой подгруппы. Решение, инвариантное относительно подгруппы $R+\alpha Z_{3}, X_{1}, X_{2}$, и решение, инвариантное относительно подгруппы $R, X_{1}, X_{2}, Z_{3}$, совпадают. Оно описывает автомодельную волну, распространяющуюся вдоль оси $O z$. Это решение имеет вид

$$
p=z^{-\frac{2}{3}} q(\xi)+\frac{1}{2}, \quad \xi=t z^{-\frac{2}{3}}
$$

Подстановка (5) в уравнение (3) дает фактор уравнение для функции $q(\zeta)$

$$
q^{m}+\left(2 q+\frac{4}{9} \xi^{2}\right) q^{\prime \prime}+2 q^{2}+2 \xi q^{\prime}+\frac{10}{9} q=0
$$

Уравнение (6) приводится к интегро-дифференциальному уравнению:

$$
\begin{aligned}
& q^{\prime}(\xi)+q^{2}(\xi)+\frac{4}{9} \xi^{2} q(\xi)=c_{2}+c_{1}\left(\xi-\xi_{0}\right)-\frac{2}{9} \int_{\xi_{0}}^{\xi} \eta q(\eta) d \eta, \\
& \text { где } \quad \xi_{0}=t_{0} z_{0}^{-\frac{2}{3}} ; c_{1}, c_{2}, t_{0}, z_{0}- \\
& \text { произвольные вещественные постоянные. }
\end{aligned}
$$

Наличие произвольных постоянных в интегро-дифференциальном уравнении (7) позволяет определить автомодельную волну, для которой в начальный момент времени $t_{0} \geq 0$ в фиксированной точке $z_{0} \neq 0$ заданы давление, скорость и ускорение его изменения: 
$p\left(t_{0}, z_{0}\right)=p_{0}, \frac{\partial p}{\partial t}\left(t_{0}, z_{0}\right)=p_{1}, \frac{\partial^{2} p}{\partial t^{2}}\left(t_{0}, z_{0}\right)=p_{2}$

где $\quad p_{0} \geq 0, p_{1}, p_{2}-$ произвольные вещественные постоянные.

В этом случае $c_{1}, c_{2}$ определяются по формулам

$c_{1}=z_{0}^{2} p_{2}+p_{1}\left(\frac{4}{9} t_{0}^{2}+z_{0}^{2}\left(2 p_{0}-1\right)\right)+\frac{5}{9} t_{0}\left(2 p_{0}-1\right)$

$c_{2}=z_{0}^{\frac{4}{3}}\left(p_{1}+\frac{1}{4}\left(2 p_{0}-1\right)^{2}\right)+\frac{2}{9} t_{0}^{2} z_{0}^{-\frac{2}{3}}\left(2 p_{0}-1\right)$.

С помощью новой неизвестной функции

$w=w(\xi)=q^{\prime}(\xi)+q^{2}(\xi)+\frac{4}{9} \xi^{2} q(\xi)$

уравнение (6) записывается в виде эквивалентной системы

$q^{\prime}=w-q^{2}-\frac{4}{9} \xi^{2} q, \quad w^{\prime}=c_{1}-\frac{2}{9} \xi u$,

для которой условия (8) принимают вид

$$
q\left(\xi_{0}\right)=z_{0}^{\frac{2}{3}}\left(p_{0}-\frac{1}{2}\right), w\left(\xi_{0}\right)=z_{0}^{\frac{4}{3}}\left(p_{1}+\left(p_{0}-\frac{1}{2}\right)^{2}\right)+\frac{2}{9} t_{0}^{2} z_{0}^{-\frac{2}{3}}\left(2 p_{0}-1\right)
$$

Ввиду гладкости правых частей системы (10) в окрестности точки $\left(\xi_{0}, q\left(\xi_{0}\right), w\left(\xi_{0}\right)\right)$, решение задачи Коши $(10),(11)$ существует и единственно в окрестности точки $\xi_{0}$. Следовательно, если константы $c_{1}, c_{2}$ заданы формулами (9), то в окрестности точки $\xi_{0}$ существует единственное решение нелинейного интегро-дифференциального уравнения (7). Подстановка этого решения в формулу (5) дает автомодельную волну, распространяюшуюся вдоль оси $O z$ и удовлетворяющую начальным условиям (8).

Аналогично устанавливается, что в окрестности точки

$\left(t_{0}, z_{0}\right),\left(t_{0}>0, z_{0} \neq 0\right)$ сушествует единственная автомодельная волна. распространяюшаяся вдоль оси $O z$ и удовлетворяющая условиям

$$
p\left(t_{0}, z_{0}\right)=p_{0}, \frac{\partial p}{\partial z}\left(t_{0}, z_{0}\right)=p_{3}, \frac{\partial^{2} p}{\partial z^{2}}\left(t_{0}, z_{0}\right)=p_{4}
$$

где $p_{0} \geq 0, p_{3}, p_{4}-$ произвольные вещественные постоянные. Эта волна определяется по формулам (5), (7) при

$$
\begin{aligned}
& c_{1}=z_{0}^{2}\left(\frac{9 z_{0}^{2}}{4 t_{0}^{2}} p_{4}+\left(\frac{27 z_{0}}{4 t_{0}^{2}}-\frac{2 t_{0}}{3 z_{0}}-\frac{3 z_{0}}{2 t_{0}}\left(2 p_{0}-1\right)\right) p_{3}+\left(\frac{1}{t_{0}^{2}}+\frac{t_{0}}{3 z_{0}^{2}}-\frac{1}{2 t_{0}}\left(2 p_{0}-1\right)\right)\left(2 p_{0}-1\right)\right), \\
& c_{2}=z_{0}^{\frac{4}{3}}\left(-\frac{3 z_{0}}{2 t_{0}} p_{3}+\left(\frac{2 t_{0}^{2}}{9 z_{0}^{2}}-\frac{1}{2 t_{0}}\right)\left(2 p_{0}-1\right)+\frac{1}{4}\left(2 p_{0}-1\right)^{2}\right) .
\end{aligned}
$$

Решение, инвариантное относительно подгруппы $R, X_{3}, Z_{3}$, описывает плоскую автомодельную круговую волну. Это решение имеет вид 


$$
p=r^{-\frac{2}{3}} q(\xi)+\frac{1}{2}, \quad \xi=t r^{-\frac{2}{3}}, r=\sqrt{x^{2}+y^{2}}
$$

Из фактор-уравнения следует, что функция $q(\xi)$ является решением следующего интегро-дифференциального уравнения:

$$
\begin{aligned}
& q^{\prime}(\xi)+q^{2}(\xi)+\frac{4}{9} \xi^{2} q(\xi)=c_{4}+c_{3}\left(\xi-\xi_{0}\right)+\frac{4}{9} \int_{\xi_{0}}^{\xi} \eta q(\eta) d \eta \\
& \text { где } \\
& \quad \begin{array}{l}
\xi_{0}=t_{0} r_{0}^{-\frac{2}{3}}, r_{0}=\sqrt{x_{0}^{2}+y_{0}^{2}} \\
c_{3}, c_{4}, t_{0}, x_{0}, y_{0}-\text { произвольные вещественные постоянные. } \\
\text { Аналогично автомодельной волне (5) устанавливается, }
\end{array}
\end{aligned}
$$
что, если в начальный момент времени $t_{0}>0$ на фиксированной окружности $r=r_{0} \neq 0$ заданы давление, скорость и ускорение его изменения

$$
p\left(t_{0}, r_{0}\right)=p_{0}, \frac{\partial p}{\partial t}\left(t_{0}, r_{0}\right)=p_{5}, \frac{\partial^{2} p}{\partial t^{2}}\left(t_{0}, r_{0}\right)=p_{6}
$$

где $p_{0} \geq 0, p_{5}, p_{6}$ - произвольные вешественные постоянные, то в окрестности этой окружности при $t$ близких к $t_{0}$ существует единственная плоская автомодельная круговая волна, удовлетворяющая начальным условиям (14). Эта волна определяется по формулам (12), (13) при

$$
\begin{aligned}
& c_{3}=r_{0}^{2} p_{6}+p_{5}\left(\left(2 p_{0}-1\right) r_{0}^{2}+\frac{2}{9} t_{0}^{2}\right)+\frac{2}{9} t_{0}\left(2 p_{0}-1\right), \\
& c_{4}=r_{0}^{\frac{4}{3}}\left(p_{5}+\left(p_{0}-\frac{1}{2}\right)^{2}\right)+\frac{2}{9} t_{0}^{2} r_{0}^{-\frac{4}{3}}\left(2 p_{0}-1\right)
\end{aligned}
$$

- Решение, инвариантное относительно подгруппы

$R, Z_{1}, Z_{2}, Z_{3}$, описывает автомодельную сферически-симметричную волну. Это решение имеет вид

$$
p=|\boldsymbol{x}|^{-\frac{2}{3}} q(\xi)+\frac{1}{2}, \quad \xi=t|x|^{-\frac{2}{3}}
$$

Из фактор-уравнения следует, что функция $q(\xi)$ является решением следующего интегро-дифференциального уравнения:

$$
\begin{aligned}
& q^{\prime}(\xi)+q^{2}(\xi)+\frac{4}{9} \xi^{2} q(\xi)=c_{6}+c_{5}\left(\xi-\xi_{0}\right)+\frac{10}{9} \int_{\xi_{0}}^{\xi} \eta q(\eta) d \eta, \\
& \text { где } \quad \begin{array}{l}
\xi_{0}=t_{0}\left|\boldsymbol{x}_{0}\right|^{-\frac{2}{3}},\left|\boldsymbol{x}_{0}\right|=\sqrt{x_{0}^{2}+y_{0}^{2}+z_{0}^{2}} \\
c_{5}, c_{6}, t_{0}, x_{0}, y_{0}, z_{0},-
\end{array}
\end{aligned}
$$

произвольные вещественные постоянные.

Аналогично автомодельной волне (5) устанавливается, что, если в начальный момент времени $t_{0}>0$ на фиксированной сфере $|x|=\left|x_{0}\right| \neq 0$ заданы давление и его радиальные производные 


$$
p\left(t_{0}, x_{0}\right)=p_{0}, \frac{\partial p}{\partial|x|}\left(t_{0}, x_{0}\right)=p_{7}, \frac{\partial^{2} p}{\partial\left|x^{2}\right|^{2}}\left(t_{0}, x_{0}\right)=p_{8}
$$

где $t_{0} \geq 0, p_{7}, p_{8}-$ произвольные вещественные постоянные, то в окрестности этой сферы при $t$ близких к $t_{0}$ существует единственная автомодельная сферически-симметричная волна, удовлетворяющая начальным условиям (17). Эта волна определяется по формулам (15), (16) при

$$
\begin{aligned}
& c_{5}=\left|\boldsymbol{x}_{0}\right|^{2}\left(\frac{9\left|\boldsymbol{x}_{0}\right|^{2}}{4 t_{0}^{2}} p_{8}+\left(\frac{27\left|\boldsymbol{x}_{0}\right|}{4 t_{0}^{2}}-\frac{2 t_{0}}{3\left|\boldsymbol{x}_{0}\right|}-\frac{3\left|\boldsymbol{x}_{0}\right|}{2 t_{0}}\left(2 p_{0}-1\right)\right) p_{7}+\left(\frac{1}{t_{0}^{2}}-\frac{t_{0}}{3\left|\boldsymbol{x}_{0}\right|^{2}}-\frac{1}{2 t_{0}}\left(2 p_{0}-1\right)\right)\left(2 p_{0}-1\right)\right), \\
& c_{6}=\left|\boldsymbol{x}_{0}\right| \frac{4}{3}\left(-\frac{3\left|\boldsymbol{x}_{0}\right|}{2 t_{0}} p_{7}+\left(\frac{2 t_{0}^{2}}{9\left|\boldsymbol{x}_{0}\right|^{2}}-\frac{1}{2 t_{0}}\right)\left(2 p_{0}-1\right)+\frac{1}{4}\left(2 p_{0}-1\right)^{2}\right) .
\end{aligned}
$$

\section{Выводы}

В нелинейной гидроакустике модель Вестервельта [1-11] используется для исследования ультразвуковых полей, генерируемых мощными излучателями. В этой модели не делается предположения о малости углов дифракции, что позволяет более точно учитывать дифракционные эффекты. Наиболее известными практическими применениями модели Вестервельта являются использование этой модели для расчета параметрических антенн сонаров и для расчета ультразвуковых полей в медицине

Для трехмерной модели Вестервельта нелинейной гидроакустики при наличии диссипации установлено, что среди инвариантных подмоделей ранга 1 эта модель имеет только три типа существенно различных (не связанных точечными преобразованиями) автомодельных волн. К ним относятся: автомодельная волна, распространяющаяся вдоль одной из осей координат, плоская автомодельная круговая волна и автомодельная сферически-симметричная волна. Получены интегро-дифференциальные уравнения, описывающие эти автомодельные волны. При некоторых условиях установлены существование и единственность таких автомодельных волн.

Наличие произвольных постоянных в полученных интегро-дифференциальных уравнениях открывает новые возможности для изучения других (отличных от приведенных в статье) имеющих физический смысл краевых задач.

Исследование выполнено при финансовой поддержке Новосибирского государственного архитектурно-строительного университета. 


\section{Библиографический список}

1. Westervelt P. Parametric acoustic array // J. Acoustic Soc. Am., 1963, vol. 35(4), pp. 535-537.

2. Novikov B.K., Rudenko V. I., Timoshenko V. I. Nonlinear underwater acoustics. New York, AIP-Press. 1987. 262 p.

3. V. A. Voronin, S. P. TarasoV, V. I. Timoshenko. Nonlinear acoustics. New York, AlP-Press. 1995. 314 p.

4. Nonlinear acoustics. Ed. By M. Hamilton and D. Blackstock. London: Academic. 1998.

5. Ostashev V.E. Acoustic in moving inhomogeneous media. London: E\&Fn Spon. 1997. 259 p.

6. Nachef S., Cathignol D., Tjotta J. N., Berg A.M., Tjotta S. Investigation of a high intensity sound beam from a plane transducer. Experimental and theoretical results // J. Acoust. Soc. Am., 1995, vol. 98 , pp. 2303-2323.

7. Tavakkoli J., Cathignol D., Souchon R., Sapozhnikov O.A. Modeling of pulsed finiteamplitude focused sound beams in time domain // J. Acoust. Soc. Am., 1998, vol. 104, pp. 2061-2072.

8. Lee Y.S. and Hamilton M.F. Time-domain modeling of pulsed finite amplitude sound beams // J. Acoust. Soc. Amer., 1995, vol. 97(2), pp. 906-917

9. Averkiou M.A., and Hamilton M.F. Nonlinear distortion of short pulses radiated by plane and focused circular pistons // J. Acoust. Soc. Amer., 1997, vol. 102(5), pp. 2539-2548.

10. Sokka S.D., King R., and Hynynen K. MRI-guided gas bubble enhanced ultrasound heating in in vivo rabbit thigh // Phys. Med. Biol., 2003 , vol. 48 , pp. 223-241.

11. Чиркунов Ю. А. Подмодели трехмерной модели Вестервельта при отсутствии диссипации // Ж.: Наука. Инновации. Технологии. 2018, вып 1. С. 81-94.

12. Чиркунов Ю. А., Хабиров С. В. Элементы симметрийного анализа дифференциальных уравнений механики сплошной среды. Новосибирск: НГТУ. 2012. 659 с.

\section{References}

1. Westervelt P. Parametric acoustic array // J. Acoustic Soc. Am., 1963, vol. 35(4), pp. 535-537.

2. B. K.Novikov, V. I.Rudenko, V. I. Timoshenko. Nonlinear underwater acoustics. AlP-Press, New York, 1987. 262 p.

3. V. A. Voronin, S. P. Tarasov, V. I. Timoshenko. Nonlinear acoustics. AIP-Press, New York, 1995. $314 \mathrm{p}$.

4. Nonlinear acoustics. Ed. By M. Hamilton and D. Blackstock. London: Academic. 1998.

5. Ostashev V.E. Acoustic in moving inhomogeneous media. London: E\&Fn Spon. 1997. 259 p.

6. Nachef S., Cathignol D., Tjotta J.N., Berg A.M., Tjotta S. Investigation of a high intensity sound beam from a plane transducer. Experimental and theoretical results // J. Acoust. Soc. Am., 1995, vol. 98, pp. 2303-2323. 
7. Tavakkoli J., Cathignol D., Souchon R., Sapozhnikov O.A. Modeling of pulsed finiteamplitude focused sound beams in time domain // J. Acoust. Soc. Am., 1998, vol. 104, pp. 2061-2072.

8. Lee Y.S. and Hamilton M.F. Time-domain modeling of pulsed finite amplitude sound beams // J. Acoust. Soc. Amer., 1995, vol. 97(2), pp. 906-917.

9. Averkiou M.A., and Hamilton M.F. Nonlinear distortion of short pulses radiated by plane and focused circular pistons // J. Acoust. Soc. Amer., 1997, vol. 102(5), pp. 2539-2548.

10. Sokka S.D., King R., and Hynynen K. MRI-guided gas bubble enhanced ultrasound heating in vivo rabbit thigh // Phys. Med. Biol. 2003, vol. 48, pp. 223-241.

11. Chirkunov Yu. A. Podmodeli trehmernoy modeli Westervelta pri otsutstvii dissipatzii (Three-dimensional model submodels Westervelt in the absence of dissipation) // J. Nauka. Innovatzii. Tekhnologii. 2018, no 1, pp. 81-94.

12. Yu. A. Chirkunov, S. V. Khabirov. Elementy simmetriynogo analiza differentzialjnyh uravneniy mehaniki sploshnoi sredy (Elements of symmetry analysis of differential equations of continuum mechanics). Novosibirsk: NGTU. 2012. 659 p.

\section{Благодарности: Исследование выполнено при финансовой поддержке Новосибирского государственного архитектурно-строи- тельного университета.}

Acknowledgments: The study was carried out with the financial support of the Novosibirsk State University of Architecture and Civil Engineering.

\section{6 авторе}

Чиркунов Юрий Александрович, д.ф.-М.н., заведующий кафедрой высшей математики Новосибирского государственного архитектурно-строительного университета. Тел.: (383) 2-66-2758.

E-mail: chr101@mail.ru.

\section{About the author}

Chirkunov Yuri Alexandrovich, Doctor of Philosophy, Head of the Department of Higher Mathematics of Novosibirsk State University of Architecture and Civil Engineering. Phone: +7(383)2-66-2758. E-mail: chr101@ mail.ru. 